\title{
AN EXPERIMENTAL STUDY ON SPINNING DISC KEY PARAMETERS INFLUENCING ITS PERFORMANCE
}

\author{
EUGENIA TEODORA IACOB-TUDOSE
}

\begin{abstract}
The spinning disc technology (SD), with its enhanced fluid flow features, has been applied to an increasing number of fields lately. Experimental characterization of the flow on a smooth or radially/concentrically indented rotating disc, for different feeding liquid flow rates $(30,50,70 \mathrm{~L} / \mathrm{h})$ and disc rotational speeds $(200,500,800,1100 \mathrm{rpm})$, using two types of feed distribution systems (one nozzle or four symmetrically distributed nozzles) has been performed, based on the pulse response technique. Based on the radial dispersion model, the Péclet number was determined for different experimental conditions and used to identify flow characteristics and to compare different spinning disc setups efficiencies. Based on the obtained experimental data, the large flowrate values and disc rotational speeds induce a flow regime closer to plug flow, however intense micromixing is achieved within an intermediate range of the studied parameters, when the turbulent wavy liquid film surface occurs, the micromixing is increased and thus, the SD technology is efficiently used. Certain flow characteristics can be achieved if the spinning disc is operated at specific parameter values.
\end{abstract}

Keywords: spinning disc, feeding system, flowrate, rotational speed, identations

\section{INTRODUCTION}

The spinning disc (SD) technology uses the centrifugal force action onto a liquid fed on a rotating disc, in order to form a thin film, characterized by intense micromixing induced by high shear rates and waves travelling on the liquid film surface [1]. Thus, enhanced transport properties [2-4] are obtained, suitable for fast gas-liquid reactions [5], homogeneous reactions and also for heterogeneous catalysis [6] or even enzymatic reactions [7] and also, for unit operations such as crystallization [8], extraction [9], boiling [10]

a "Gheorghe Asachi” Technical University of lasi, „Cristofor Simionescu” Faculty of Chemical Engineering and Environmental Protection, Department of Chemical Engineering, 73 Prof. Dr. Docent D. Mangeron Blvd., 700050, lasi, Romania, e-mail: eugenia.iacob2017@gmail.com 
or condensation [11]. A large number of applications such as food, cosmetic and pharmaceutical product manufacturing $[12,13,14]$, polymer production $[15,16]$, treatment of industrial wastewaters for the degradation of some pollutants [17,18,19], nanoparticle manufacturing $[20,12,21]$ prove the high versatility of the spinning disc technology.

This experimental study was initiated in order to explain some of the results obtained using SD technology in a laboratory setup in textile wastewater treatment [19] and to investigate key parameters influencing the disc performance. The residence time distribution (RTD) based on the pulseresponse method was applied in the liquid film on a spinning disc, at different liquid flow rates and disc rotational speeds, with different liquid feeding systems (one orifice of different diameters and respectively, four symmetrically distributed orifice system), for a smoothed and respectively indented surface disc, in order to render information regarding the radial dispersion and also, the most suitable configurations to increase the SD technology efficiency.

The RTD can be a useful tool to investigate the mixing performance of a SD system, giving information related to the system's degree of dispersion (or Péclet number, high dispersion means low Péclet number, usually $\mathrm{Pe}<10$ [22]) and also, allows comparisons regarding the efficiency of different experimental setups.

Some of the literature data suggest that depending on the feeding system used for liquid inlet on the disc, the convection structures of the liquid film are different which influences, in the end, the SD effectiveness. Specifically, if the liquid film is fed through the center of the disc than concentric waves are formed, developing in spiral waves towards the edge of the disc [23], while a liquid stream fed through one nozzle, above the disc induces standing waves formation [24] and also, spiral waves occurrence. The lastly mentioned setup was investigated in this work, using different diameter single orifice or four symmetrically distributed orifice systems in order to assess its effect on dispersion.

Furthermore, studies regarding the characteristics of the flow on a spinning disc found that large liquid flowrates and rotational speed values, low viscosity liquids would render a convection on the spinning disc closer to the plug flow [25, 26]. However, experimental data [19] obtained on SD technology applied in textile wastewater treatment indicate that a flow regime closer to plug flow is not always appropriate for attaining the best SD efficiency. The degree of dispersion and whether the plug flow is attained in our system can be revealed by calculating the Pe numbers for different working conditions from the RTD experimental data. 
The diffusion equation for the tracer used flowing on the spinning disc is:

$$
\frac{\partial C}{\partial t}=D \frac{\partial^{2} C}{\partial r^{2}}-u \frac{\partial C}{\partial r}
$$

Using some initial and boundary conditions for an open-open system and solving the above Equation (1), the normalized residence time distribution function $E(\theta)$ is obtained, with the normalized variance, $\sigma_{\theta}^{2}$, given by $[27,28]$ :

$$
\sigma_{\theta}^{2}=\frac{2}{P e}+\frac{8}{P e^{2}}
$$

where $\mathrm{Pe}$ is the Péclet number. Based on the experimental value $\sigma_{\theta}^{2}$, using Equation (2), one can calculate the Pe number.

The tracer concentration obtained experimentally and the Equations (3) to (8) have been used to render respectively, the residence time distribution function, $E(t)$, the normalized residence time distribution function, $E(\theta)$, the mean residence time, $\tau$, the normalized mean residence time, $\theta$, the variance, $\sigma^{2}$ and the normalized variance, $\sigma_{\theta}^{2}$, as follows [28]:

$$
\begin{aligned}
& E(t)=\frac{C(t)}{\int_{0}^{\infty} C(t) d t} \\
& \tau=\frac{\int_{0}^{\infty} t C(t) d t}{\int_{0}^{\infty} C(t) d t} \\
& \sigma^{2}=\frac{\int_{0}^{\infty} t^{2} C(t) d t}{\int_{0}^{\infty} C(t) d t}-\tau^{2} \\
& E(\theta)=\tau E(t) \\
& \theta=\frac{t}{\tau} \\
& \sigma_{\theta}^{2}=\frac{\sigma^{2}}{\tau^{2}}
\end{aligned}
$$

where $\mathrm{C}(\mathrm{t})$ is salt concentration at time t. Randomly, for a few liquid flow rates and disc rotational speeds, the salt concentration time variation was checked in order to confirm a good reproducibility of the results.

\section{RESULTS AND DISCUSSIONS}

Residence time distribution curves can be symmetrical and narrow in the conditions of an ideal plug flow, in radial direction. In this case, dispersion is negligible. The more the flow deviates from this type of ideal convection, the more asymmetric and dispersed the residence time distributions. 


\section{One nozzle feeding system - influence of the nozzle diameter and liquid flowrate for smooth surface disc}

Figure 1 (a)-(d) shows the normalized residence time distributions, $\mathrm{E}(\theta)$, calculated at three different water flow rates of $10,30,50 \mathrm{~L} / \mathrm{h}$, for four constant disc rotational speeds of $200,500,1100,2000 \mathrm{rpm}$, respectively, for a feeding inlet nozzle of $3.5 \mathrm{~mm}$ diameter. Absolutely all distributions have a characteristic, relatively symmetrical shape. At constant rotational speed, an increase in the flow rate from $10 \mathrm{~L} / \mathrm{h}$ to $70 \mathrm{~L} / \mathrm{h}$ renders slightly slender curves, indicating less dispersion, in agreement to Mohammadi's findings [25]. The longer tails, obtained for $\theta>1$, suggest the persistence of the liquid on the spinning disc, fact confirmed experimentally by other literature studies that used flow visualization and found liquid dragging on the disc surface [24]. Similar curves for the normalized residence time distribution function were obtained for nozzles of $2.45 \mathrm{~mm}$ and respectively, $1.95 \mathrm{~mm}$ diameters, presented in figures 2 and 3, respectively, for three different liquid flow rates of 10,50 and $70 \mathrm{~L} / \mathrm{h}$, at constant disc rotational speed of 200 and respectively, $2000 \mathrm{rpm}$.
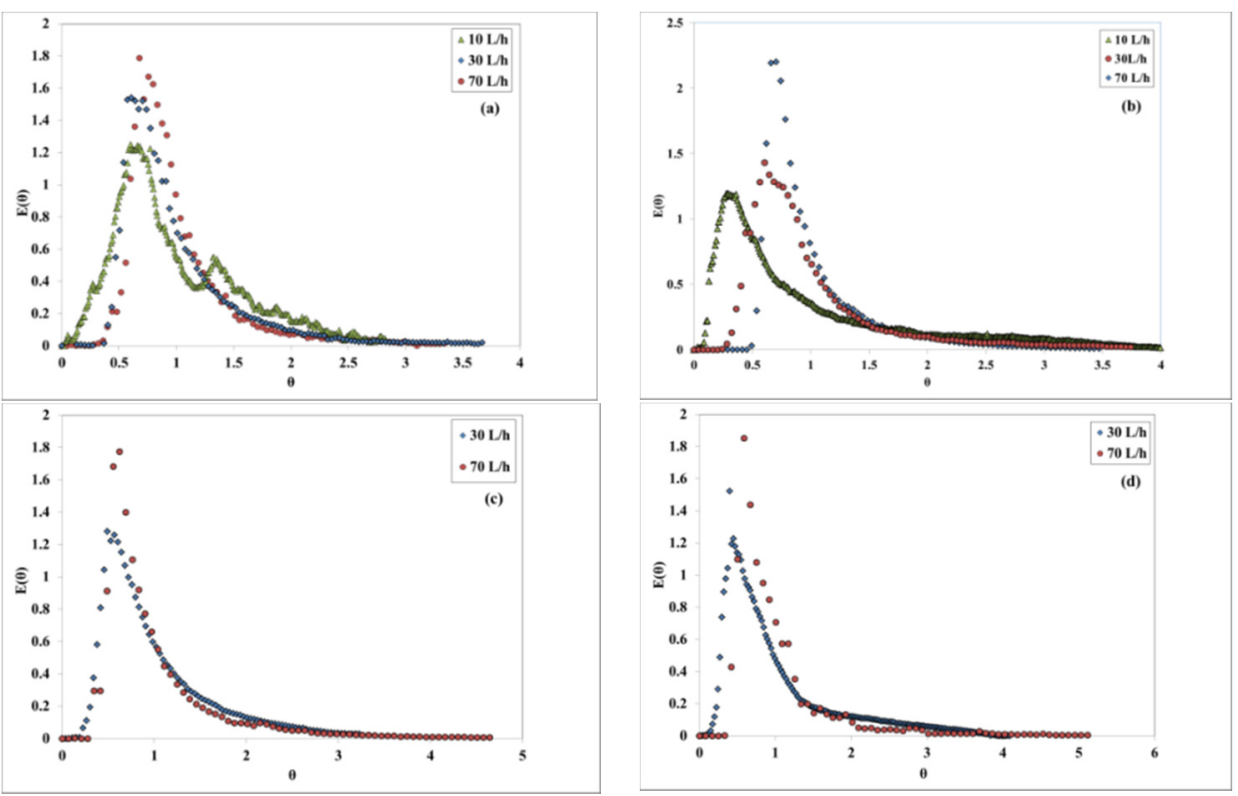

Figure 1. Influence of the liquid flowrate, at constant disc rotational speed, nozzle diameter ø 3.5 mm, smooth disc: (a) 200 rpm; (b) 500 rpm; (c) 1100 rpm; (d) 2000 rpm 

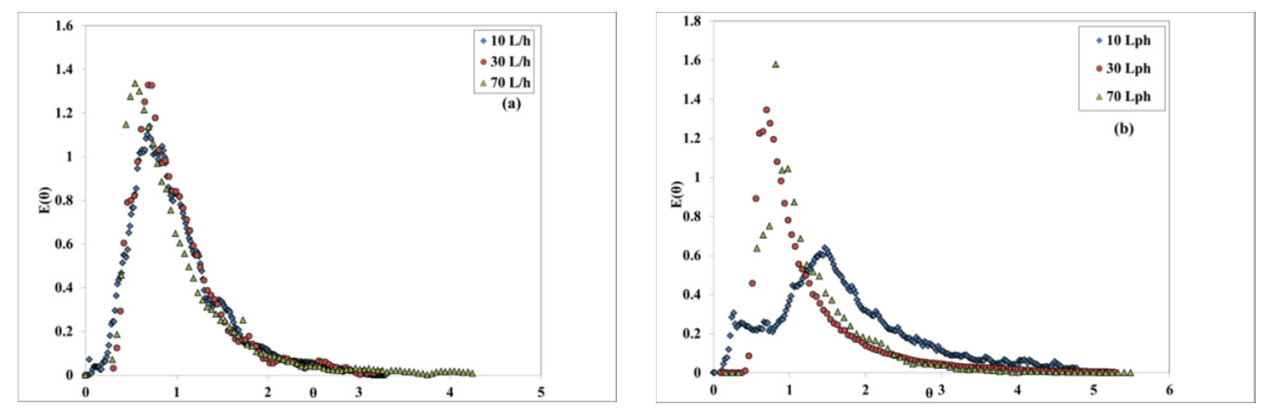

Figure 2. Influence of the liquid flowrate, at constant disc rotational speed, nozzle diameter ø 2.45 mm, smooth disc: (a) 200 rpm; (b) 2000 rpm
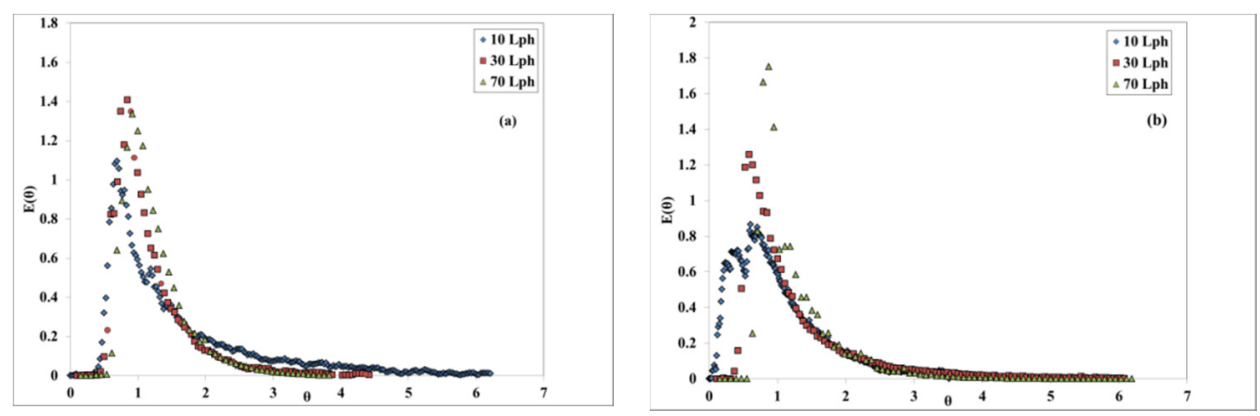

Figure 3. Influence of the liquid flowrate, at constant disc rotational speed, nozzle diameter ø 1.95 mm, smooth disc: (a) 200 rpm; (b) 2000 rpm

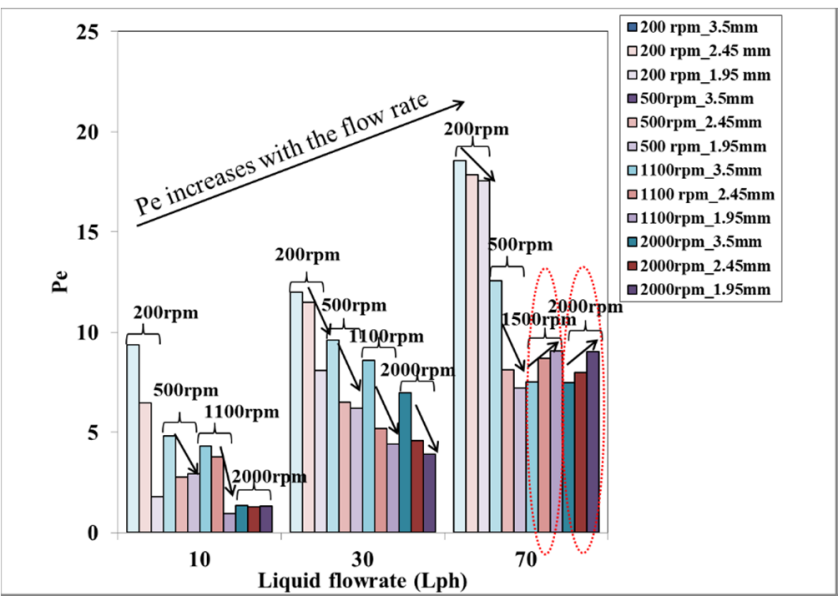

Figure 4. Comparison of different diameter feeding orifices with ø3.5, $\varnothing 2.45$ and $\varnothing 1.95 \mathrm{~mm}$ on the spinning disc - smooth surface (Pe calculated based on the radial dispersion model) 
A comparison between different orifice nozzle diameters, based on figures 1,2 and 3 is difficult to make, thus, from the calculated normalized variance, $\sigma_{\theta}^{2}$, using equation (8), the Péclet number was determined based on equation (2) and represented in figure 4 for different flowrates and disc rotational speeds.

The larger the orifice feeding diameter, the larger the Péclet number at lower flow rates of 10 and $30 \mathrm{~L} / \mathrm{h}$, at all investigated rotating speed ranging between $200 \mathrm{rpm}$ and $1100 \mathrm{rpm}$. Smaller orifice diameter means larger velocity of the liquid jet on the spinning disc, thus, more dispersion on its surface. This result is similar to some other studies regarding a jet impinged, however, on an axially moving surface and found to experience greater dispersion at larger surface speeds [29]. Nevertheless, at $70 \mathrm{~L} / \mathrm{h}$, the trend is the same for the low range of rotational speed values of 200 and $500 \mathrm{rpm}$, but at larger rotating speeds of 1100 and $2000 \mathrm{rpm}, \mathrm{Pe}$ increases with the decrease of the orifice diameter, probably due to atomization occurrence which renders a lower liquid flow rate.

\section{Four symmetrically distributed nozzle feeding system}

\subsection{Influence of the liquid flow rate and disc rotational speed}

Figure 5(a)-(d) shows the normalized residence time distributions, $E(\theta)$, calculated at three different water flow rates of $30,50,70 \mathrm{~L} / \mathrm{h}$, for four constant disc rotational speeds of $200,500,800,1100 \mathrm{rpm}$, respectively.
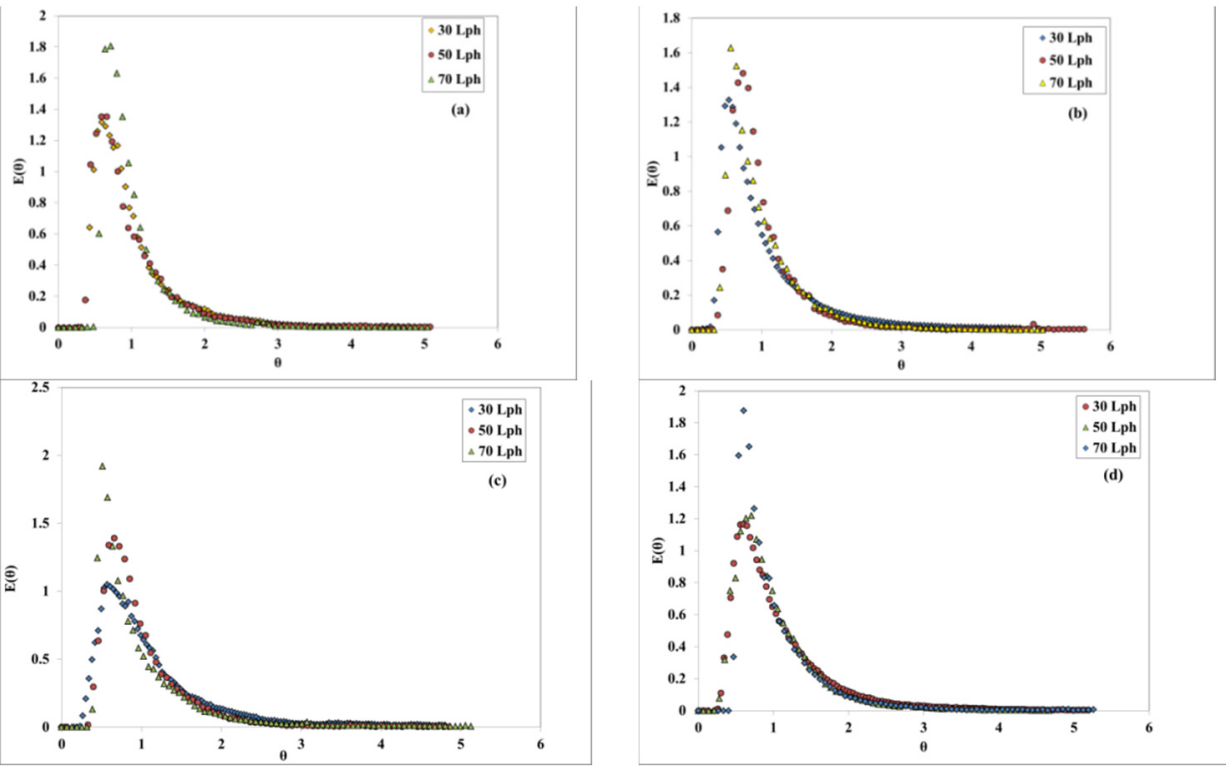

Figure 5. Influence of the liquid flowrate, at constant disc rotational speed, smooth disc: (a) $200 \mathrm{rpm}$; (b) $500 \mathrm{rpm}$; (c) 800 rpm; (d) 1100 rpm; four-point feeding system $\varnothing 1.75 \mathrm{~mm}$ 
One can observe for the four symmetrically distributed orifice feeding system of $1.75 \mathrm{~mm}$ diameter (the four nozzles of $\varnothing 1.75 \mathrm{~mm}$ have an equal area with the one $ø 3.5 \mathrm{~mm}$ nozzle) similar residence time distributions dependencies on liquid flow rate and disc rotational speed to the ones obtained with one nozzle feeding system. A comparison between the two equal flow areas feeding systems did not reveal significant differences, even though it is thought that the symmetrically distributed system should provide a more symmetric flow field on the disc than the one orifice system.

\subsection{Influence of the disc surface}

All the data presented to this point pertain to a smoothed spinning disc surface. The residence time distributions obtained on a radially and respectively, concentrically indented discs and comparisons to the smoothed surface disc are included in the following section.

\subsubsection{Disc surface with radial indentations}

The indentations on a surface are known to promote turbulence. The spiral movement of the liquid on the spinning disc hinted towards the use of a radially indented disc (Figure $10(\mathrm{a})$ ) that might promote additional mixing in comparison to a smooth surfaced disc, as long as the liquid flow on the disc does not have a strong radial component. Figure 6 (a), (b) shows the normalized residence time distribution obtained for a radially grooved disc, at three different flow rates of 30,50 and $70 \mathrm{~L} / \mathrm{h}$ and constant disc rotational speeds of 200 and $1100 \mathrm{rpm}$. For the grooved disc, similar distributions $E(\theta)$ in time and their dependence on the liquid flow rate, namely narrower curves for higher liquid flow rates, can be observed as for the smooth disc.
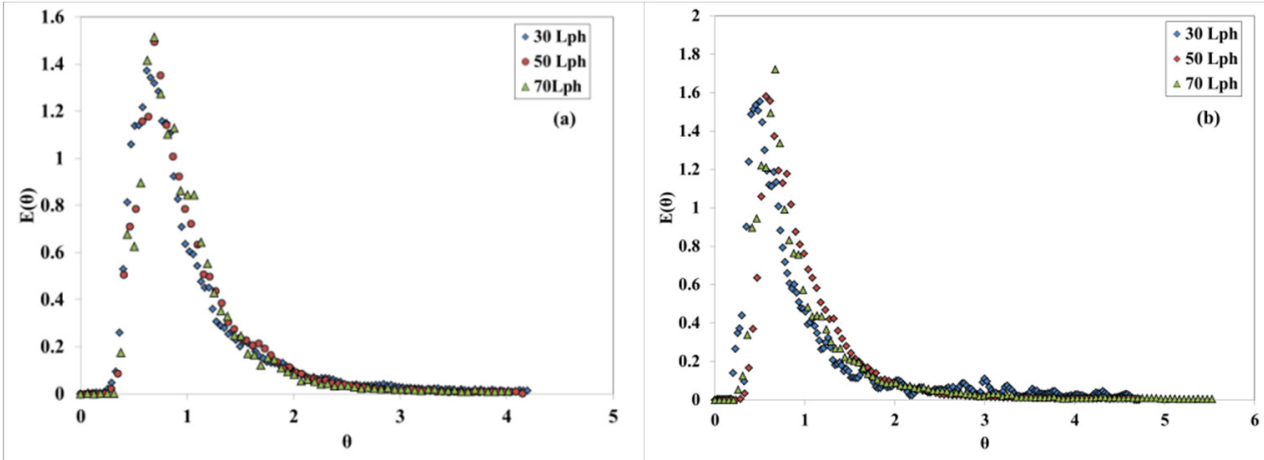

Figure 6. Influence of the liquid flowrate, at constant disc rotational speed, radially indented disc: (a) $200 \mathrm{rpm}$; (b) $1100 \mathrm{rpm}$. 
In order to compare the dispersion for the two surfaces, the Péclet number was calculated from Eq. (2), based on the normalized variance, $\sigma_{\theta}^{2}$, determined from experimental data, using Eq. (8). Figure 7 presents a comparison between the Pe numbers, for both smooth and radially grooved discs, at different liquid flow rates and rotational speeds. For liquid flow rates of 30 and $50 \mathrm{~L} / \mathrm{h}$, at all the investigated rotational speeds, the Pe number was slightly lower for the smooth disc than for the radially grooved disc, which indicates that the dispersion is lowered and the radial micromixing is enhanced for the indented disc.

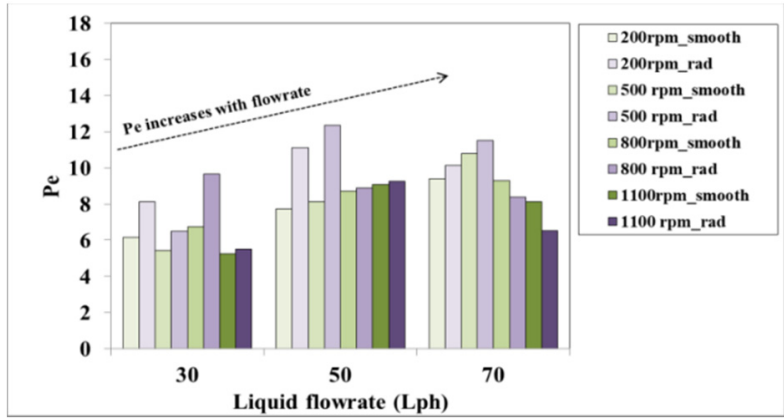

Figure 7. Comparison between smooth and radially indented discs based on calculated Pe number

At a liquid flow rate of $70 \mathrm{~L} / \mathrm{h}$, this trend was similar for rotational speeds of 200 and $500 \mathrm{rpm}$, while at 800 and $1100 \mathrm{rpm}$, the trend changed probably due to a combined increased liquid flow rate and rotational speed that possibly induced drainage through the radial grooves of the disc (the red dashed circles). Also, Figure 7 reveals an increased Pe number as the liquid flow rate increases, for most of the investigated rotational speeds, for both smooth and radially discs, which is consistent with the narrower residence time distribution curves obtained. Concluding the comparison between the two discs, one can use the radially indented disc to enhance the micromixing, especially at low to moderate rotational speeds and all investigated liquid flow rates.

\subsubsection{Disc surface with concentric indentations}

The concentrically indented disc should also promote intense micromixing, thus the disc of $20 \mathrm{~cm}$ with five concentrical grooves (Figure 10(b)) was used to obtain the residence time distribution. Figure 8 indicates that the plots have similar dependencies on the investigated liquid flow rates of 30,50 and $70 \mathrm{~L} / \mathrm{h}$, at constant rotational speeds of 200 and $1100 \mathrm{rpm}$, as previously discussed. 

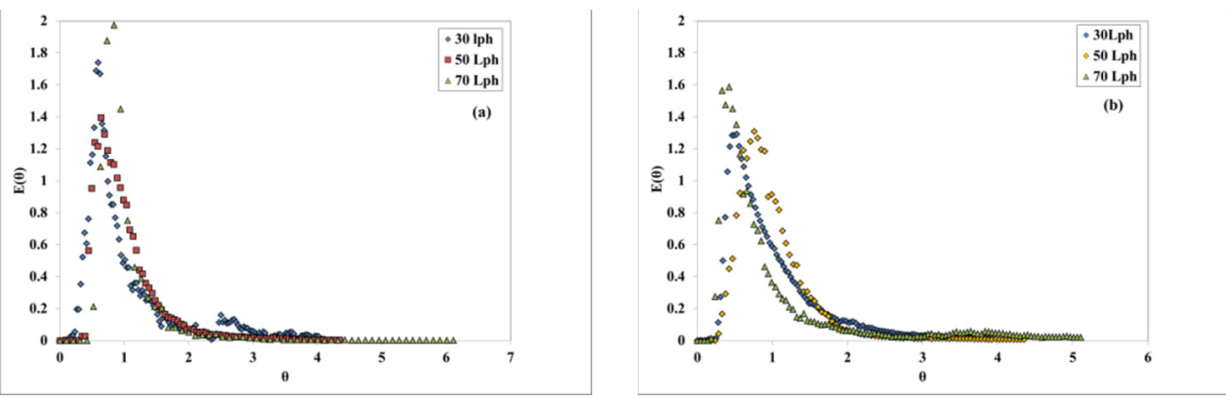

Figure 8. Influence of the liquid flow rate, at constant disc rotational speed, concentrically indented disc: (a) 200 rpm; (b) 1100 rpm

Comparison between the calculated Pe numbers using Eq.(2) (based on the normalized distribution, $\sigma_{\theta}^{2}$, calculated with Eq.(8) from experimental data), for both smooth and concentrically grooved discs, at different liquid flowrates and rotational speeds are presented in figure 9 . For 30 and $50 \mathrm{~L} / \mathrm{h}$ liquid flowrates, the Pe number based on axial dispersion model for the grooved surface is slightly larger than for the smooth surface, for all investigated disc rotational speeds of $200,500,800$ and $1100 \mathrm{rpm}$. These results are similar to Mohammadi's findings [25] that revealed, using the RTD analysis and the tanksin-series model, at disc rotational speeds of 300 and $1200 \mathrm{rpm}$ and a liquid flowrate of $15 \mathrm{~mL} / \mathrm{s}$ (i.e. $54 \mathrm{~L} / \mathrm{h}$ ), that the concentrically indented disc is characterized by a larger number of tanks-in-series than a smooth surface disc, thus generates more micromixing. For this study, at larger liquid flowrates of 70 $\mathrm{L} / \mathrm{h}$, a similar trend is valid for 200,500 and $800 \mathrm{rpm}$, however, at $1100 \mathrm{rpm}$, the trend is reversed, probably due to the atomization occurrence, which reduces the liquid flowrate on the discs (the red dashed circle in figure 9). In this case, the use of a concentrically indented disc promotes also more turbulence than the smoothed surface disc.

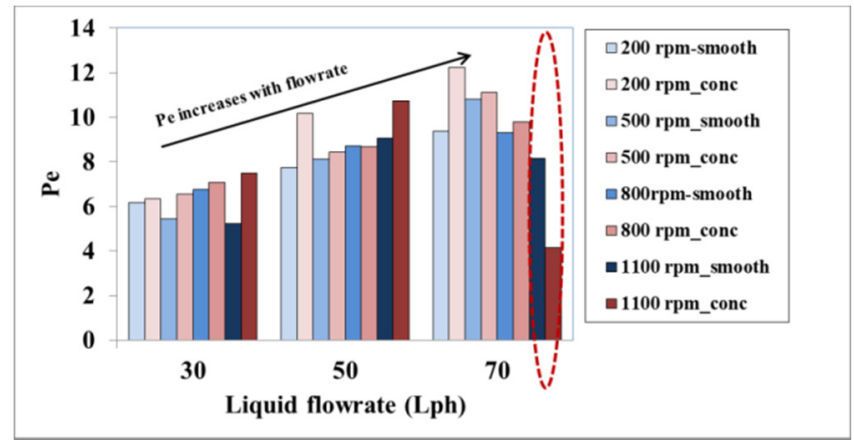

Figure 9. Comparison between smooth and concentrically indented discs based on calculated Pe number 
A comparison between the radially and concentrically indented discs, regarding their efficiency in promoting turbulence is difficult to make, even though the Pe numbers for the radially grooved disc seem somewhat higher than for the concentrically grooved disc, however for both discs the Pe values lie within a range indicating mixed flow.

\section{CONCLUSIONS}

The pulse response technique was applied in order to characterize some of the flow features on a spinning disc of $20 \mathrm{~cm}$ diameter, with different water feeding systems, for different liquid flowrates and disc rotational speeds, in smooth and also indented, both radially and concentrically, disc setups. The experimental data indicated that for a smoothed surface disc, an increase in the liquid flow rate $(10,30,70 \mathrm{~L} / \mathrm{h})$, at constant rotational speeds $(200,500,1100$ and $2000 \mathrm{rpm}$ ), causes a reduction in the dispersion. An increase in the disc rotational speed, within the low range (<800 rpm), induces a flow regime with less dispersion. However, within the high range values, atomization is prevalent and the RTD results can no longer be used. Furthermore, the chosen feeding system can induce less dispersion on the disc if larger diameter orifice is used. The Péclet numbers for the radially grooved disc, investigated for the first time, and also, for the concentrically grooved disc, are higher than for the smooth surface disc, especially in the low to moderate disc rotational speeds and for all investigated flow rates. Experimental data obtained at low flowrate and disc rotational speed values, using a smaller orifice diameter of the feeding system and a smoothed surface disc, indicate more dispersion, otherwise, the disc flow may be getting closer to a plug flow, even though the largest obtained $\mathrm{Pe}$ numbers in our system suggest still a reasonable amount of dispersion. These observations point out that that operating the SD within specified conditions would render certain flow characteristics, appropriate for the studied system.

\section{EXPERIMENTAL SECTION}

The experimental setup used to obtain all the experimental data is presented in Figure 10, together with detailed pictures of the radially (a) and respectively, concentrically (b) grooved discs. It consists of a spinning disc 3 driven by a variable speed, adjustable motor 5 through the coupling 4 . The disc is placed in a case 6 , provided with a cooling chamber 7 in case the liquid overheats. The tap water used in the experiment was supplied on the rotating disc via a metal rod 2 , with one nozzle of different diameters (ø3.5, 2.45 and $1.95 \mathrm{~mm}$ ) or four symmetrically distributed nozzles, each of $\varnothing 1.75$ $\mathrm{mm}$ diameter, from tank 10 , using a pump 8 , through flowmeter 9 , which 
measured the liquid flow rate, maintained constant by adjusting tap11. The spinning disc exit liquid was collected through a special machined piece which accommodated the conductometer probe 12, connected to the precalibrated conductivity meter 13 (Conductivity Meter 14, WTW 315i).
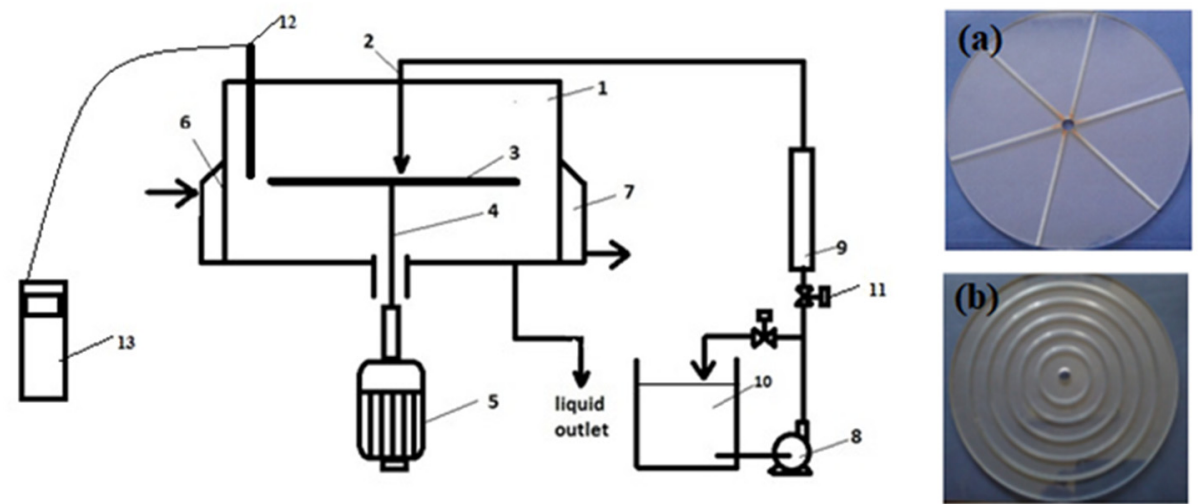

Figure 10. Experimental setup with spinning disc (a) radial grooves (b) concentric grooves

The disc rotational speed was measured using a laser tachometer with a precision of $\pm 0.1 \mathrm{rpm}$.

Once the steady state operating regime was reached, the pulse response technique was applied. It consisted of a $2 \mathrm{ml}$ sodium chloride $(\mathrm{NaCl})$ solution, $0.5 \mathrm{~mol} / \mathrm{L}$, injection directly into the disc inlet water jet within 1s. At the same time, the conductance values were recorded with a frequency of $1 \mathrm{~Hz}$ until the displayed value reached the very initial conductance value. Based on the calibration curve, previously obtained, the $\mathrm{NaCl}$ concentrations at the disc exit were calculated.

\section{REFERENCES}

1. A.I. Stankiewicz; J.A. Moulijn; Chem. Eng. Prog., 2000, 96, 22-34.

2. R.J.J. Jachuck; C. Ramshaw; Heat Recov. Syst.CHP, 1994, 14(5), 475-491.

3. I.S. Boiarkina; Norris, D. Patterson, Chem. Eng. J., 2013, 225, 752-765.

4. L-B Yao; W. Wu; X.-S. Wu; G.-W. Chu; Y. Luo; B.-C. Sun, Chem Eng Process, 2021, 166, 108500-108521. 
5. A. Chaudhuri; K.P.L. Kuijpers; R.B.J. Hendrix; P. Shivaprasad; J.A. Hacking; E.A.C. Emanuelsson; T. Noël; J. van der Schaaf, Chem. Eng. J., 2020, 400, 125875.

6. M. Vicevic; Jachuck R.J.J.; K.Scott, J.H. Clark; K. Wilson, Green Chem, 2004, 6, 533-537.

7. X. Feng; D.A. Patterson; M. Balaban; G. Fauconnier; E.A.C. Emanuelsson, Chem. Eng. J., 2013, 221, 407-417.

8. L. Cafiero; G. Baffi; A. Chianese; R. Jachuck, Ind Eng Chem Res, 2002, 41(21), 5240-5246.

9. F. Visscher; J. van der Schaaf; M.H.J.M.de Croon; J.C.Schouten, Chem. Eng. J., 2012, 185-186, 267-273.

10. M.M. de Beer; J.T.F. Keurentjes; J.C. Schouten; J. van der Schaaf, AIChE J., 2016, 62 (10), 3763-3773.

11. M.M. de Beer; J.T.F. Keurentjes; J.C. Schouten; J. van der Schaaf, AIChE J., 2016, 62 (10), 3784-3796.

12. S. Sana; K. Boodhoo; V. Zivkovic, Green Process Synth, 2019, 8, 507-515.

13. W.H. Khan; V.K. Rathod, Chem. Eng. Proc., 2014, 80, 1-10.

14. P. Oxley, C. Brechtelsbauer, Francois Ricard, Norman Lewis Industrial \& Engineering Chemistry Research 39(7), 2000, 2175-2182.

15. K.V.K. Boodhoo; R.J. Jachuck; Green Chem., 2002, 2, 235-244.

16. K.V.K. Boodhoo; R.J. Jachuck; Appl. Therm. Eng., 2000, 20(12), 1127-1146.

17. C.Y. Chang; N.L. Wu, Ind. Eng. Chem. Res., 2010, 49, 12173-12179.

18. A.Y. Zhang; M.H. Zhou; L. Han; Q.X. Zhou, J. Hazard. Mater., 2011, 186, 13741383.

19. E.T. lacob-Tudose; C. Zaharia, Appl Sc, 2020, 10(23), 8687.

20. C. Ahoba-Sam, V.K. Boodhoo, U. Olsbye, K.J. Jens, Materials, 2018, 11, 154.

21. M. Stoller, J.M. Ochando-Pulido, Nanomaterials, 2020, 10, 1321.

22. Levenspiel, O. Chemical reaction engineering, 3, John Wiley \& Sons, New York, USA, 1999, p.259.

23. Woods, W. The hydrodynamics of thin liquid films flowing over a rotating disc, Ph.D.thesis, University of Newcastle upon Tyne, UK, 1995.

24. Boiarkina, I.S. Investigation of a Spinning Disc as a Thin Film Photocatalytic Reactor for the Degradation of Recalcitrant Wastewaters, Ph.D. thesis, The University of Auckland, 2013.

25. Mohammadi, S., Boodhoo, K.V.K., Chem. Eng. J., 2012, 207-208, 885-894.

26. Boodhoo, K.V.K., Al-Hengari, S.R., Chem. Eng. Technol., 2012, 35(7), 12291234.

27. Bozga, G., Muntean, O. Reactoare chimice (Chemical reactors), 1, Ed.Tehnica, Bucuresti, Romania, 2000, p.169.

28. Coulson, J.M., Richardson, J.F. Chemical Engineering, 3, 3, Elsevier Butterworth-Heinemann, Oxford, UK, 2007, p.90.

29. Moulson, J.B.T., Green, S.I., Phys. Fluids, 2013, 25(10),102-106. 